\title{
Database Agile Management Dependent on MIS Infrastructure
}

\author{
Masaru Furukawa \\ TOYAMA University
}

frukawa@eco.toyama-u.ac.jp

\begin{abstract}
Today agility of management cannot be realized unless given all of the following three kinds of quickness: a) quick recognition of environmental changes, b) quick decision-making on countermeasures against these changes, and c) quick implementation of the countermeasures chosen. In the business world nowadays the quickness of a) concerns information that an MIS deals with, the quickness of b) concerns use of an MIS for decisionmaking, and the quickness of c) concerns change of an MIS itself. With a focus on c) and assuming that flexibility is a core property that an MIS should acquire to realize agile management, our study aims to clarify how MIS flexibility should be enhanced. With this aim in mind, we will survey the sources and types of change demands on an MIS and consider in a structured way the characteristics that an MIS should have, such that they will conduce to its flexibility in dealing with these change demands. Having laid the groundwork mentioned so far, we will discuss practical methods to enhance MIS flexibility, and end by presenting our evaluation procedure for MIS flexibility.
\end{abstract}

Keywords: Management Information Systems, MIS Flexibility, MIS Evaluation, MIS Infrastructure.

\section{Introduction}

Most leading companies worldwide are increasing IT (information technology) investment in order to achieve competitive edges in the rapidly changing management environment. This trend has made the evaluation of MIS (management information systems) more crucial and indispensable than ever. What a company expects from IT investment is quick acquisition of value and/or effectiveness that an MIS will bring to its business. Therefore today realization of agile management is recognized as the most important keyword in the business world.

This agility can only be realized given all of the following three kinds of quickness: a) quick recognition of an environmental change, b) quick decision-making on countermeasures against the change, and c) quick implementation of the countermeasures chosen. In the business world nowadays the quickness of a) concerns information that an MIS deals with, the quickness of b) concerns use of

Material published as part of this proceedings, either on-line or in print, is copyrighted by the author with permission granted to the publisher of Informing Science for this printing. Permission to make digital or paper copy of part or all of these works for personal or classroom use is granted without fee provided that the copies are not made or distributed for profit or commercial advantage AND that copies 1) bear this notice in full and 2) give the full citation on the first page. It is permissible to abstract these works so long as credit is given. To copy in all other cases or to republish or to post on a server or to redistribute to lists requires specific permission from the author. an MIS for decision-making, and the quickness of c) concerns change of an MIS itself.

With a focus on c) above and assuming that flexibility is a core property that an MIS should acquire to realize agile management, our study aims to clarify how MIS flexibility should be enhanced. With this aim in mind, we will survey the sources and types of change demands on MIS, and we will consider in a structured way the characteristics that an MIS should have, such that they will conduce to its flexibility in dealing with these change demands. Having laid the groundwork mentioned so far, we will discuss practical methods to enhance MIS flexibility, and end by presenting our evaluation procedure for MIS flexibility.

\section{Definition of the Problems and Angles of Analysis}

\section{Relationship between MIS Value and Agile Management}

We might give the following paraphrases for the six kinds of MIS value defined in "Information Economics (Parker, 1988)" in relation to agile management:

- Value of investment: It means economic utility an MIS produces for the organization concerned. Quickness of capital recovery (or high rate of turnover) will allow the organization greater business profits. 
- Value of management information: It means utility an MIS generates by providing in the form of management information the critical success factor (CSF) affecting the success or failure of the business. Quicker acquisition of information required for decision-making when coping with environmental changes will enable the organization to take quicker action against the changes.

- Value for competition: It means utility an MIS generates by enabling the organization concerned to catch up with a competitor with a business advantage over it. Obviously, if poorer MIS efficiency on its part continues, it will only aggravate the business disadvantage it sustains against the competitor. Here the urgent challenge for the organization is to transform its MIS into one as good or preferably better than that of the competitor.

- Value of strategy support: It means utility brought about by the use of an MIS as a support for the current business strategies. In this context, generally MIS implementation will be accompanied by changes in both SOPs (standard operating procedures) and the MIS itself. Quick implementation of these changes will boost the efficiency in the accomplishment of the current business strategies.

- Value of competitive edge: It means utility an MIS offers by producing new managerial strategies, new products and/or methods to defeat its competitor and/or get over barriers confronting it. If the competitor is going to take equivalent strategies, you cannot have a competitive edge over them unless you take action earlier than they.

- Value of MIS infrastructure: MIS infrastructure consists of hardware, software, data and communication network. It is a prerequisite for the generation of other MIS values since its renovation will affect the efficiency of an MIS in generating these other values. Since it is impossible to run a business these days without an MIS, we can assume that a project to change SOPs virtually means a planned change in the MIS and that, given a properly renovated MIS infrastructure, the project will require far less cost and time than otherwise.

That is to say, agility of management cannot be realized unless well-renovated MIS infrastructure guarantees efficient MIS change requiring minimum cost and time. The business value of an MIS (hereafter MIS value for short) is generated by the use process of an application function working on MIS infrastructure (Hamillton, 1981). Then the value of an MIS might be represented by the following formula (drawing on similar formulas in the literature (Johanson, 1993; Hanaoka, 1997)):

$$
V=\frac{f(F, U)}{g(C, T)}
$$

where $C, T, F$ and $U$ stand for cost, time, function and use, respectively.

\section{Present State of MIS Evaluation}

As for evaluation of MIS effectiveness, methods traditionally utilized have been, in the classification of cost/benefit methodology, "Total Quantification with Qualitative analysis (UGIS, 1981)", "Information Economics" and " Contribution to Corporate Performance" (Utunomiya, 1993; Myer, 1989). But perception and use of a particular information system can be heavily conditioned by personal and situational variables (Lucas, 1974). This fact in particular makes it difficult to evaluate MIS effectiveness quantitatively. Deemed relatively reliable for this purpose, however, are the following five measures: "High levels of system use", "User satisfaction with the system", "Favorable attitudes about MIS function", "Achievement of objectives", "Financial payoff" (Laudon, 2000). In fact, many MIS researchers have shifted their focus to the human and organizational measures of system success such as information quality, system quality, and the impact of systems on organizational performance (DeLone, 1992).

These evaluation methods focus on the numerator of formula (1), which in effect represents the MIS use process, i.e. how easily adaptable an MIS is for the user. Quick use of an adapted MIS enables a) quick recognition of an environment change, and b) quick decision-making on countermeasures against the change. However, for all the research efforts on this adaptability, we know of no established methods that an organization can use to maximize MIS utility (i.e. the numerator of formula (1)).

On the other hand, c) quick implementation of countermeasure chosen to cope with environmental changes involves change of an MIS itself. These days MISs have been growing increasingly large in scale, and so has the demand for modification of existing ones to cope with rapid changes inside and outside organizations. Unfortunately, however, we have no systematized methods we can turn to in order to minimize the cost and time required to meet change demands (i.e. the denominator of formula (1)). 


\section{Database Agile Management Dependent on MIS Infrastructure}

We hear of many cases of MIS implementation that have met with troubles such as failure to deliver by the due date, excess over the estimates, productivity deterioration (increase of backlogs), malfunctioning (activity inability, operational inability, increase of bugs), system failure (failure of a system to be used as intended). All this shows that no reliable methods have been established to estimate the denominator of formula (1) (i.e. the cost and time for MIS implementation).

\section{Definition of the Problems}

There are many possible problems demanding detailed analysis concerning both the denominator and the numerator of formula (1). Basic angles of analysis on each category might be as follows:

- The denominator of formula (1) can be interpreted in two ways. One interpretation is that quick acquisition of MIS value can be achieved by shortening of the cycle time (quick decision-making, high rate of turnover, etc.) and the other is that quick acquisition of MIS utility will be achieved by quick change of an application function working on MIS infrastructure. Fundamentally we can expect these achievements to be accelerated greatly by technological innovation, although they are affected to some extent by the characteristics of the organization concerned.

- On the other hand, the numerator of formula (1) can be interpreted to mean "the use of the application function working on MIS infrastructure". To understand the mechanism of how this is achieved, analysis should be focused on organizational behavior in relation to the MIS.

Considered in light of this structural classification of the problems, the literature published in recent years includes pretty many works focused on the numerator but few on the denominator. Therefore in this study, we will confine our analysis to the denominator side. The denominator is represented by cost and time as the penalty of change for the change of an MIS function. Hereafter, we will use the abbreviated notations of "POC" and "MIS flexibility" to represent the penalty needed for MIS change and the facility of an MIS to absorb change demands, respectively.

The smaller the $P O C$, the greater are the agility and the flexibility with which an organization can execute a change of the MIS required to cope with environmental changes affecting management. Formula (2) is transformed from formula (1) to represent MIS flexibility (Flex) on the assumption that the numerator of the latter formula is constant (Const).

$$
\text { Flex }=\frac{\text { Const }}{g(C, T)}=\frac{1}{P O C}
$$

Formula (2) suggests that $P O C$ can serve as a substitute index for quantitative evaluation of the flexibility of an MIS. It also obviously shows the following relationship between MIS flexibility and $P O C$ :

- If $P O C$ is high, MIS flexibility is low.

- If $P O C$ is low, MIS flexibility is high.

Accordingly, let us define MIS flexibility as follows:

MIS flexibility serves as an index for measurement of the ability to absorb future demands for MIS change, and can be accounted for in terms of cost and time.

The aim of this paper is to elucidate in a structured way the characteristics of MIS flexibility and derive strategies to expand it.

\section{Structure of MIS Flexibility}

In order to discuss our present theme in more practical terms, let us translate our definition of MIS flexibility into the following question:

"How flexible an MIS should we acquire at the time of its implementation so as to able to accommodate possible changes in the future as they occur?"

Henceforward let us consider MIS flexibility from this viewpoint.

\section{Change demand on MIS}

When an MIS division undertakes a change of the MIS, the change demand is likely to have originated from the following three kinds of sources:

- Changes in external environment: Policy changes by governmental agencies, e.g. implementation of environment accounting, change of the consumption tax rate, etc. These changes typically demand accomplishment by the appointed time.

- Changes of internal business functions: Changes of management strategies and/or SOPs aimed at enhancement of ROI (return on investment), 
Masaru Furukawa

enhancement of management information, support for management strategies and/or acquisition of a competitive edge. These changes differ in urgency and importance depending on their aims.

- IT renovation: Changes demanded by the MIS division itself aimed at better service for the MIS user, e.g. implementation of CASE (computer-aided system engineering) and/or change of a DBMS (data base management system), etc. These are indispensable for securing both effective maintenance of an existing MIS and preparedness to meet potential change demands in anticipation.

An MIS division cannot cope with these changes unless it has already secured adequate management resources: e.g. computer throughput, storage and long-term serious efforts by experienced engineers, etc. In actuality, however, MIS divisions often fail to have an adequate reserve of resources required. Thus we find frequent occurrence of MIS divisions' failure to meet an entire change demand by the appointed time. This, in turn, leads to a delay in MIS change and the execution of a management strategy and/or a BP (business process) change, and obstructs the realization of agile management. An MIS division cannot cope with MIS change demands agilely as they occur unless they have predicted potential change demands in advance and provided adequate resources and well-renovated MIS infrastructure.

\section{Meanings of the Renovation of MIS Infrastruc- ture}

An MIS is a system consisting of hardware and software. A change or a modification of this system entails risks (e.g. system breakdown). If these risks are realized, the system incurs cost and time before it is restored. Table 1 lists changes of a system, risks of a change, and well-known ITs available for use in evasion strategies against risks.

It is a matter of common knowledge that proper application of IT to MIS infrastructure lowers not only change risks but also the $P O C$ (cost and time) for future system development, i.e. it facilitates subsequent system developments. But IT application itself incurs a high $P O C$, which acts as a self-limiting factor against it. Moreover, an MIS division might not be able to obtain enough resources from the management to execute MIS infrastructure renovation unless they have a well-established method for evaluation of ROI involving the renovation. This accounts for the reluctance of many MIS divisions to venture to undertake MIS infrastructure renovation.

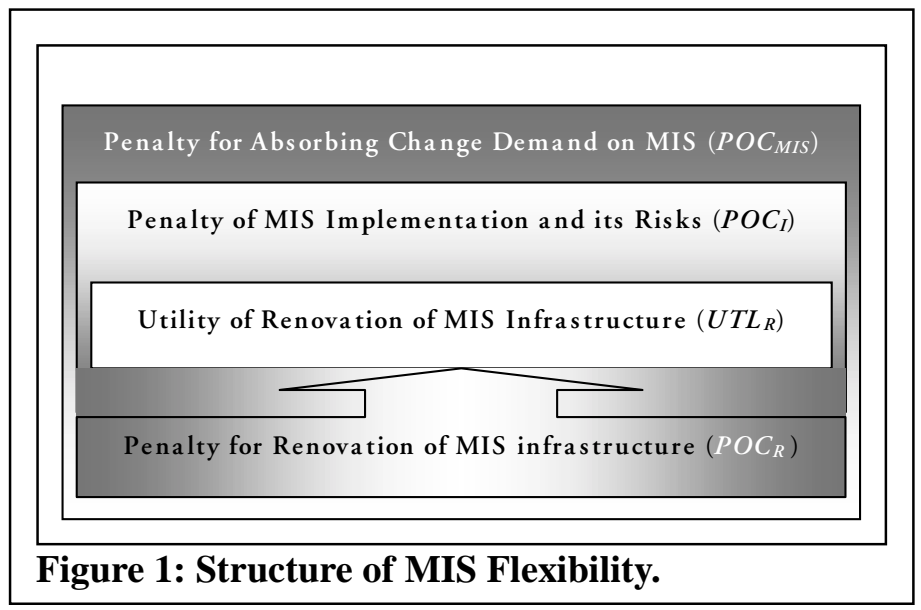

Here let us take a brief look at the mission of an MIS division. Obviously its major role is to dispose of demands for change of an existing MIS originating from internal and external changes. The division is called upon to perform this mission efficiently: i.e., at a minimum cost and in a minimum time. This in effect means that it should only pay a minimum $P O C$ to get the system well enough organized to absorb future change demands.

In actual system development, it is known that time shortening in the midst of a project can incur the following risks, which are likely to cause system failures.

- An increase in the urgency of a project due to time shortening renders the project structure unstable, and increases project risks: i.e., if the system requirements are clear and straightforward, the project will be highly structured, if not, it will be fluid and unstable. (Yin, 1974).

- An additional deployment of engineers for time shortening can be counter-productive since it increases management cost, and actually leads to a slowdown in the project. (Laudon, 1994).

Means of time shortening of a system project, therefore, should be provided not after the start of the project, but before. More specifically, it is realistic to consider that the aim of time shortening can be achieved by moderate renovation of the infrastructure of an existing MIS in advance of the project (e.g., standardization of the development method and the system structure, application of CASE and/or DBMS).

A moderate renovation of MIS infrastructure can ensure greater ease and efficiency in MIS modification [utility of MIS infrastructure renovation]. It is therefore another mission of an MIS division to vigorously promote renovation of MIS infrastructure from a long-term perspective. Here- 


\section{Database Agile Management Dependent on MIS Infrastructure}

after, when we talk about the application of IT to an existing MIS for the purpose of enhancing MIS flexibility, let us use the expression "renovation of MIS infrastructure".

But implementation of infrastructure renovation incurs a $P O C$ of its own as well as facilitating MIS modification. Here, let us represent this fact in the whole structure of MIS flexibility in terms of the substitute index of $P O C$ as in Figure 1. Figure 1 suggests that the $P O C$ paid for a moderate renovation of MIS infrastructure can have the benefit of reducing the $P O C$ required to cope with future change demands [utility of renovation] $\left(U T L_{R}\right)$. This means that renovation of MIS infrastructure is moderate application of IT for evasion of change risks accompanying MIS implementation.

Thus the $P O C$ of a whole MIS change $\left(P O C_{M I S}\right)$ can be represented formulaically as follows using the abbreviations shown in Figure 1:

$$
\begin{gathered}
P O C_{M I S}=P O C_{I}+\left(P O C_{R}-U T L_{R}\right) \\
\text { Planning Procedure } \\
\text { Focused on MIS Flexibility }
\end{gathered}
$$

\section{Changes of an MIS}

In order to discuss our present theme in more practical terms, let us translate our definition of MIS flexibility into the following question:

"How flexible an MIS should we acquire at the time of its implementation so as to be able to accommodate possible changes in the future?"

To give an answer to this question, we need to be able to predict future change demands likely to be made on an existing MIS before considering how to deal with them. Here let us break down change demands into two groups, one originating from outside an MIS and the other arising from inside and consider the characteristics that MIS flexibility should maintain in relation to these two kinds of change demands.

\section{Characteristics Required of MIS to cope with Change Demands (External Factors)}

Change demands on an MIS must be predicted separately for each kind of demand source. If we are to cope with all predicted change demands, we need to execute capacity requirement planning (CRP).

For proper implementation of CRP planning, we need to see to the following:

- System alternatives: available methods for disposal of each change demand,

- Estimate of management resources: volume of resources (computer power, volume of storage, manpower, etc.) required to execute each alternative,

- Capacity for change demands: volume of resources available for disposal of change demands.

Below we will explain in concrete terms the three external characteristics that an MIS should maintain (hereafter to be referred to as "external factors"):

- Product: This is an ability to build a system capable of creating various application functions with the same pieces of equipment. In the short term, this means that the system has the adaptability to the fluctuating volumes of different change requests in the sense that it is capable of dealing with each change request economically and promptly. In the long term, it means that several application functions can utilize the same system equipment while generating higher returns on investment by extending its life cycle. This life cycle extension is constrained by the internal characteristics.

- Volume: This is the facility of a system to absorb the quantitative fluctuation of change demands within the bounds of the budget, that is to say, the facility to cut down or expand its own scale. Its validity as a characteristic of MIS flexibility is easily understandable seeing that the development and maintenance of an MIS have something in common with order production in manufacturing systems. This ability is also constrained by the internal characteristics.

- Working sequence: This ability has to do with the handling of the working sequence of various kinds of hardware, data and operations, which affects the creation of application functions. This ability is constrained by the flexibility of the structure of the system itself: i.e. by the internal characteristics. This characteristic means that the system will be able to continue service to its user, even if the system suffers a partial breakdown and/or the operator is absent.

As to these characteristics, one important aspect we should focus our analysis on is "the volume of change demands that must be absorbed". Since disposal of external change demands resembles order production in manufacturing systems, the planning method of CRP in manufacturing 
Masaru Furukawa

systems is applicable to MIS planning as a means of absorbing maximum change demands with an existing capacity. However, we must note that there are fundamental differences between development of an MIS system and order production in manufacturing systems as shown bellow:

- In CRP for MIS implementation, the unit of manmonth alone, which has traditionally been used as a unit of production capacity, cannot do justice to the POC required for MIS implementation (Brooks, 1974), since MIS implementation depends more on experience and skill of engineers than on equipment used for development. Therefore our CRP should also include these human factors as planning factors.

- An MIS is a system made up of many subsystems, but MIS flexibility depends not so much on how each of the subsystems is developed as on how well renovated the infrastructure of the whole system is (MIS infrastructure is one of our core concepts, to which we will devote detailed discussion in the subsequent subsection).

Further, in the evaluation of the ability to absorb change demands, because of their great effects on the $P O C$ and the quality of the finished system, the following must be taken into consideration: the "availability" of hardware, the "structure" of an existing MIS, "experience and knowledge" about the service area of a subsystem that is going to be built and the technologies that are going to be chosen. All these have to do with the characteristics of an MIS itself, which will affect the relative difficulty of MIS change (internal factors). These will also be discussed in detail in the next subsection.

\section{Change Risks and their Evasion Strategies (Internal Factors)}

An MIS consists of hardware such as computers with basic

\begin{tabular}{|c|c|c|c|c|}
\hline \multicolumn{2}{|c|}{$\begin{array}{l}\text { Cate } \\
\text { gory }\end{array}$} & Meaning & Change with Risk $=>$ Risk & Evasion Strategy \\
\hline \multirow{5}{*}{ 总 } & \multirow{2}{*}{ 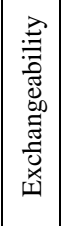 } & \multirow{2}{*}{$\begin{array}{l}\text { Easiness of exchange } \\
\text { and change of hard- } \\
\text { ware }\end{array}$} & $\begin{array}{l}\text { Failure in machine replacement }=>\text { System unus- } \\
\text { able }\end{array}$ & $\begin{array}{l}\text { Enhancement of Connection interchangeability, Enhancement of } \\
\text { Upper compatibility (open protocol, open system) }\end{array}$ \\
\hline & & & $\begin{array}{l}\text { Failure in upgrading of basic software }=>\text { System } \\
\text { unusable }\end{array}$ & $\begin{array}{l}\text { Enhancement of Connection interchangeability, Enhancement of } \\
\text { Upper compatibility, Multiplexing, back up \& recovery, insurance \& } \\
\text { maintenance contract, out-sourcing (external equipment) }\end{array}$ \\
\hline & \multirow{3}{*}{$\frac{0}{9}$} & \multirow{3}{*}{$\begin{array}{l}\text { Ability to continue } \\
\text { service of giving appli- } \\
\text { cation functions }\end{array}$} & $\begin{array}{l}\text { Bugs in basic software => System uncontrollable, } \\
\text { System breakdown }\end{array}$ & Back up \& recovery, preventive maintenance \\
\hline & & & $\begin{array}{l}\text { Bugs in application programs }=>\text { System unusable, } \\
\text { System failure }\end{array}$ & $\begin{array}{l}\text { Thoroughness of testing, standardization, Educational training, Back } \\
\text { up \& recovery }\end{array}$ \\
\hline & & & Mistake in operation $=>$ System failure & Educational training, Job enrichment, Outsourcing (skilled engineer) \\
\hline \multirow{4}{*}{ 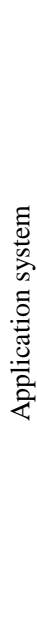 } & \multirow{2}{*}{ 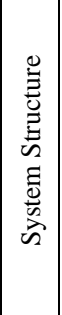 } & \multirow{2}{*}{$\begin{array}{l}\text { Ability to add new } \\
\text { application function } \\
\text { (degree of structuring) }\end{array}$} & \multirow{2}{*}{$\begin{array}{l}\text { Environment change of outside, Enterprise function } \\
\text { change => Due date delay, Excess over the esti- } \\
\text { mates, Productivity deterioration, Malfunction, } \\
\text { System failure }\end{array}$} & $\begin{array}{l}\text { Technological viewpoint: Standardization of protocol (open system) } \\
\square \text { Structured analysis / design / programming, and Data Oriented } \\
\text { Approach (Structuring, Normalization) }\end{array}$ \\
\hline & & & & $\begin{array}{l}\text { Organizational viewpoint: Experience of an engineer and en- } \\
\text { hancement of skill, educational training of a user, Workload } \\
\text { (reduction of overload of an engineer), Job enrichment, Practical use } \\
\text { of external consultant }\end{array}$ \\
\hline & 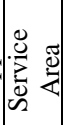 & $\begin{array}{l}\text { Ability to supply inex- } \\
\text { perienced service for } \\
\text { the first time }\end{array}$ & $\begin{array}{l}\text { Inexperienced field => Due date delay, Excess over } \\
\text { the estimates, Productivity deterioration, Mal- } \\
\text { function, System failure }\end{array}$ & Rearranging Entity and Building database \\
\hline & 官 & $\begin{array}{l}\text { Ability to supply a } \\
\text { service with inexperi- } \\
\text { enced technology } \\
\text { and/or method }\end{array}$ & $\begin{array}{l}\text { IT innovation, Introduction of new technology }=> \\
\text { Due date delay, Excess over the estimates, Produc- } \\
\text { tivity deterioration, Malfunction, System failure }\end{array}$ & $\begin{array}{l}\text { Accumulation of experience, } \mathrm{R} \& \mathrm{D}, \text { Standardization of system devel- } \\
\text { opment, Educational training (dissolution of skill deficiency) }\end{array}$ \\
\hline
\end{tabular}

Table 1 $\square$ System-Risk and IT 


\section{Database Agile Management Dependent on MIS Infrastructure}

software built into it, storage, communication equipment and software such as programs and data for business use.

A typical change of hardware occurs where there is a need for 1) an exchange for expansion of the capacity to cope with entire change demands, or for 2) an exchange for expansion of the availability to reduce the probability of system breakdowns. Let us refer to the former as "exchangeability", the latter as "fault tolerance".

On the other hand, a change of application systems occurs where there is a need to change an MIS in order to execute business strategies. This kind of change differs in difficulty depending on whether it involves the "system structure (including the data structure)" of the existing system, or the "service area" and "IT adoption" new to an MIS division.

Below, in order to define the internal characteristics, let us first consider the sources and risks of changes, the evasion strategies for risks, and the evaluation methods for these changes. These are listed in Table 1.

Here, let us consider how internal factors should be viewed in realistic terms. In an urgent system change, restoration of the system has priority over the consideration of how to minimize of the $P O C$ of the change. Accordingly it is realistic to consider that evaluation of internal factors should be focused on the variety of possible combinations of strategy alternatives it has for immediate disposal of a change demand. $P O C$ (i.e. cost and time) is considered as a common coordinate axis on which to evaluate each internal factor. To illustrate this line of thinking, Table 2 lists change factors and their evaluation indices.

In formula (3), $P O C_{I}$ means the risk of change, $P O C_{R}$ means the $P O C$ of risk evasion strategy, and $U T L_{R}$ means the utility of risk evasion strategy.

\section{Easiness of Hardware Change}

\section{Exchangeability}

Definition: The easiness of exchanging or modifying computer hardware, network, basic software, e.g., exchange and increase of computers (including manufacturer change), changes from mainframe to workstation, enlargement of memory storage, changes of communication equipment, etc.

\begin{tabular}{|c|c|c|c|c|c|}
\hline \multirow{2}{*}{\multicolumn{2}{|c|}{ 递 }} & \multicolumn{4}{|c|}{ Index for Evaluation } \\
\hline & & Viewpoint & Cost & Time & Utility \\
\hline \multirow{2}{*}{ 胥 } & 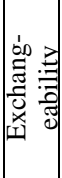 & $\begin{array}{l}\text { Enhancement of Connec- } \\
\text { tion interchangeability, } \\
\text { Enhancement of Upper } \\
\text { compatibility (open } \\
\text { protocol, open system) }\end{array}$ & $\begin{array}{l}\text { Human } \\
\text { resource } \\
\text { (Man- } \\
\text { month) }\end{array}$ & $\begin{array}{l}\text { Time dis- } \\
\text { tance } \\
\text { (exchange } \\
\text { speed) }\end{array}$ & $\begin{array}{l}\text { Shortening } \\
\text { of exchange } \\
\text { time, reduc- } \\
\text { tion of cost }\end{array}$ \\
\hline & 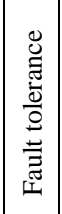 & Availability & $\begin{array}{l}\text { Opportu- } \\
\text { nity loss, } \\
\text { Recover- } \\
\text { ability cost }\end{array}$ & $\begin{array}{l}\text { MTBT, } \\
\text { MTTR }\end{array}$ & $\begin{array}{l}\text { Reduction } \\
\text { of opportu- } \\
\text { nity loss } \\
\text { and recov- } \\
\text { erability } \\
\text { cost }\end{array}$ \\
\hline \multirow{4}{*}{ 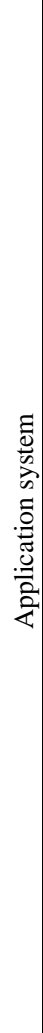 } & & $\begin{array}{l}\text { Structuring of System } \\
\text { and program }\end{array}$ & $\begin{array}{l}\text { Cost for } \\
\text { change } \\
\text { demand, } \\
\text { Cost for } \\
\text { structuring }\end{array}$ & $\begin{array}{l}\text { Time for } \\
\text { change } \\
\text { demand, } \\
\text { Time for } \\
\text { structuring }\end{array}$ & $\begin{array}{l}\text { Reduction } \\
\text { rate of } P O C \\
\text { for design }\end{array}$ \\
\hline & 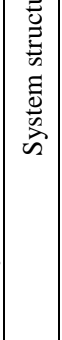 & $\begin{array}{l}\text { Degree of database } \\
\text { (Number of access path } \\
\text { from application pro- } \\
\text { gram to data, Number of } \\
\text { program and data that } \\
\text { should be changed, Rate } \\
\text { of entity implemented in } \\
\text { database), Tendency of } \\
\text { backlog volume on time } \\
\text { axis }\end{array}$ & $\begin{array}{l}\text { Cost for } \\
\text { change } \\
\text { demand, } \\
\text { Cost for } \\
\text { database } \\
\text { develop- } \\
\text { ment }\end{array}$ & $\begin{array}{l}\text { Time for } \\
\text { change } \\
\text { demand, } \\
\text { Time for } \\
\text { database } \\
\text { develop- } \\
\text { ment }\end{array}$ & $\begin{array}{l}\quad \text { Tabl } \\
\text { Reduction } \\
\text { rate of } P O C \\
\text { for design }\end{array}$ \\
\hline & 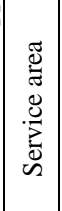 & $\begin{array}{l}\text { Rate of BP and entity } \\
\text { given a service }\end{array}$ & $\begin{array}{l}\text { Cost for } \\
\text { change } \\
\text { demand, } \\
\text { Cost for } \\
\text { new ser- } \\
\text { vice }\end{array}$ & $\begin{array}{l}\text { Cost for } \\
\text { change } \\
\text { demand, } \\
\text { Cost for } \\
\text { new service }\end{array}$ & $\begin{array}{l}\text { Reduction } \\
\text { of } P O C \text { for } \\
\text { design }\end{array}$ \\
\hline & 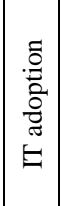 & $\begin{array}{l}\text { Technical continuity and } \\
\text { degree of experience }\end{array}$ & $\begin{array}{l}\text { Cost for } \\
\text { change } \\
\text { demand, } \\
\text { Cost based } \\
\text { on leaning } \\
\text { degree }\end{array}$ & $\begin{array}{l}\text { Time for } \\
\text { change } \\
\text { demand, } \\
\text { Time based } \\
\text { on leaning } \\
\text { degree }\end{array}$ & $\begin{array}{l}\text { Reduction } \\
\text { of } P O C \text { by } \\
\text { learning }\end{array}$ \\
\hline
\end{tabular}

Cause of change: A change of hardware means an exchange of hardware itself or an upgrade of componential basic software. The aim of this exchange is to expand capacity to cope with change demands, i.e. enhancement of system availability (reduction of risk of system breakdown).

Risk of change: These changes entail risks of system unavailability due to failure in exchange or excess over the estimated time for exchange. 
Risk evasion strategy: Since realization of the risks causes damage $(P O C)$ to an MIS before it is restored, it is necessary to provide risk evasion strategies to minimize potential damage. One such strategy is to standardize protocol to secure connectivity and upper compatibility with new hardware to be installed. Other effective evasion strategies for facilitating urgent exchange might be outsourcing of equipment, maintenance contract and insurance. These strategies will enhance hardware exchangeability by reducing the $P O C$ for hardware exchange.

Evaluation: In evaluation of this aspect of flexibility, we need to estimate the $P O C$ for future exchanges, and the $P O C$ for risk evasion strategies and their utility (i.e. how much $P O C$ for potential future exchanges they could reduce). In other words, we need to predict when and how often we need to exchange componential hardware comprising the existing MIS, list exchange methods, and estimate the $P O C$ for each of the exchange methods in terms of the man-day (cost and time) required. Risk evasion strategies include standardization of protocol, maintenance contract, etc. Reduction of risks by a combination of these strategies can be regarded as the utility of these strategies and needs to be estimated as such.

Relationship with external factors: Facilitation of exchange of hardware makes it possible to provide demanded application functions in the form of a variety of "products". Facilitation of a combination of hardware items will make it easier to change "working sequence". Accordingly, high exchangeability enhances the external flexibility factors of "product" and "working sequence".

Example: Instead of having the exchangeability of automaton components constrained by protocols peculiar to hardware manufacturers, MAP (manufacturing automation protocol) in industry enhanced the flexibility factor of exchangeability by limiting the purchase of automaton components to products designed in compliance with the protocol prescribed by users. Thus this protocol contributed greatly to the reduction of the cost and the enhancement of the quality of an automaton. On the other hand, the low exchangeability of online terminals in early 90 's led to the formation of a high barrier to foreign airlines' access to U.S. aviation industry and had a seriously adverse effect on enterprise management as seen in the case of the CRS (computer reservation system) of American Airlines.

\section{Case 1: Preparation for the New Millennium}

System designers ought to have been able to foresee the occurrence of the year 2000(Y2K) problem at the stage when the data were being designed. This even implies that they virtually programmed the Y2K problem, which they could have averted.

Corporation $\mathrm{X}$ was one of the first corporations in Japan that have introduced computers. They also very early undertook a change in their application system from batch to on-line real time processing. The change was executed by adding DAM files (direct access method) and programs written in Assembler for real-time processing to the existing batch processing system. The new system was only used during the daytime. The old batch system took over data from the new system after regular office hours for processing during the night. A scrap-and-build approach to the system development had been dismissed in order to meet the demand of the executives, who were anxious to start using the new system as soon as possible. In 1988, the MIS Division of the firm was very busily occupied with maintenance of the system that had been built 20 years before, and was swamped with a huge backlog. After racking their brains about how to overcome their predicament, they decided to replace an old DB with a relational database (RDB). The procedure that they worked out for the change consisted of:

- $\quad$ building a new RDB normalized with a data dictionary $(D D)$, with all data from the existing MIS integrated into it,

- creating an interface between the existing MIS and the new $R D B$,

- $\quad$ and finally switching over from the existing MIS to the new system, which would access the new RDB directly.

This renovation cost far more than expected and required serious efforts of the engineers. But both the running cost and the backlog decreased as the changeover progressed. In the fall of 1999, most IT personnel in the world were in great fear of the arrival of the Y2K. At this time, the changeover of the firm's MIS had already been completed. Because of the superior flexibility of the MIS infrastructure (system structure), the expansion of the date-fields to accommodate the change of millennia was completed by the next day by a mere modification of the definition of the date-fields in the DD.

To build a DB with a DD, it is indispensable to carry out the definition of key fields and their relationship, which incurs $P O C$ of its own. But properly created, a DD will bring us the following utilities: the ease of data use, which enhances the agility of decision-making on selection of a strategy alternative for coping with environment change, the ease of MIS renovation, which enhances the agility of the execution of the selected action.

\section{Fault Tolerance}

Definition: the ability to promptly restore a system while continuing service of an application function when confronted with a partial or total system breakdown (a change of a system itself). Tolerance for fault in this sense is made possible by the provision of a functional substitution in advance.

Cause of change: A system breakdown can be attributed to a) defective and malfunctioning hardware, b) a failure in 


\section{Case 2: Change of SOPs}

Building a new information system is a form of planned organizational change involving many different people. Since this sort of socio-technological change involves changes in work, management and structuring of the organization, we should take good account of the following as many authors of MIS literature have been suggesting (Parker, 1988; Pneuell, 1997; Richardson, 1980):

- difficulties involved in management change,

- $\quad$ fitting technology to the organization (or vice versa),

- $\quad$ and understanding the limitations of IT $\square$

Kanban-system is well known worldwide as a typical Japanese production control system. But actually many Japanese corporations had traditionally adopted MBPN (management by production number), and some of them attempted to implement an MRP without success. The Robot system section of the corporation Y attempted business process re-design in 1984 and 1988.

The quantity of robot order had sprung from one or two to more than 10 per month between 1982 and 1983, and a further order expansion had been predicted. They prefabricated common units and parts of a robot system for which the order was virtually settled. After final specification decision, these and other customer options were assembled into a finished product before its shipment. The order expansion, however, generated backlogs of unused parts, which led to a rise in production costs. It was this predicament that faced them with the need to re-design the management process.

Corporation Y believed that the application of MRP could provide a specific solution for the problem, and tried its application twice. The first attempt ended in an utter failure (i.e. it turned out to be unusable), and the second attempt was successful to the extent that the developed system proved to be at least partially usable. There was a marked difference in system effectiveness between the first and the second attempt. It could be attributed to the following managerial considerations in the second attempt.

replacement of basic software or a software bug, c) a bug in an application system and d) operation error.

Risk of change: a) and b) lead to risk of system uncontrollability, c) to system uncontrollability or a system failure and d) to a system failure.

Risk evasion strategy: Strategies for evasion of each of these risks include, for a), multiplexing, back up \& recovery, insurance $\&$ maintenance contract, out-sourcing of equipment, for b), back up \& recovery, preventive maintenance, for c), standardization affecting system development, thoroughness of testing, educational training, back up \& recovery, and, for d), educational training, job enrichment, out-sourcing of skilled engineers.

Evaluation: Evaluation of this aspect of flexibility should be focused on system availability. Generally a combination of MTBF (mean time between failures) and MTTR (mean time to repair) is used as an evaluation index. As the groundwork for evaluation, we need to list the causes of system breakdowns, to keep their occurrence frequency
- $\quad$ avoiding unnecessary use of computers: creating a structure in which production activity was adequately and smoothly achieved by independent decisions of workers, i.e. rearranging the work order in the production line, thoroughness of visual control, the practice of placing materials in proper order.

- $\quad$ seeing to moderation in organizational changes: step-by-step implementation of the new system in order to minimize the degree of change, i.e. a step-by-step application of the production number MRP system.

This brings home to us the following:

- Human beings are far more flexible than an MIS,

- If the changes adopted do not exceed the tolerance of the organization, they will more likely be accepted.

In order to attain a goal for administrative change, an organization may have to go through several steps successively. We have seen that the length of a step (in a change) must not exceed the tolerance of an organization. The MRP system package did not have enough flexibility for a planned change not to overload the tolerance. On this account the package required extra costs for the addition of the MBPN function (since after this re-design project, all Japanese computer manufacturers have developed MRP systems that include the MBPN function). MBPN-MRP has become a standard production control system in Japan. An MBPN-MRP system permits an organization to carry out a step-by-step system implementation, and is more flexible than a standard MRP system. When an organization undertakes a step-by-step change, the MIS should have enough flexibility (system structure) to fit in with the change process.

To build an MIS as a combination of modules, it would be advantageous to adopt the method of structured analysis/design. However, there should be a trade-off between the implementation effort and organizational resistance against the change and the POC that it would incur. One possibly advantageous option would be to design an aggregate of objects, when planning step-by-step implementation of MIS function in line with the progress of organizational learning.

under observation for each type, and estimate the $P O C$ of their future occurrence. The $P O C$ for risk evasion strategies can be evaluated in terms of cost and time required for a combination of IT implementations. The utility of risk evasion (i.e. $P O C$ reduction of a future system breakdown) can be evaluated in terms of potential reduction of opportunity loss and recovery cost.

Relationship with external factors: Enhancement of system availability enhances the external flexibility factor of "volume", which in turn, enhances the flexibility of "working sequence".

Example: System configurations such as dual, duplex and tandem, and fault-tolerant computers are among the results of the use of reliability engineering to improve the fault tolerance (system availability) of a whole system. 


\section{Easiness of Application System Change}

\section{System Structure}

Definition: This factor involves the ability to create new application functions with a combination of hardware and software and network to cope with demands for system development or system modification. A well-structured MIS (which means that the system is built of a combination of modules) can change its application functions or add to them by changing only some modules since the target modules are independent of other modules (This flexibility factor also includes securing of the independence of data from the program and building of a database in the sense of data structuring). Let us refer to this kind of flexibility as "system structure".

Cause of change: This flexibility factor involves change of application functions, which occurs when business planning requires a change of the existing MIS.

Risk of change: Risks are built into the system in the process of its development. They include failure to deliver by the due date, excess over the estimates, productivity deterioration (increase of backlogs), malfunction (system uncontrollability, increase of bugs, etc.), and system failures in the sense that the system cannot be used as intended. These risks are generated by deficiency in requirement definition and design, engineers' deficiency in experience and skill, inadequate educational training for users, etc. If realized, these risks can obstruct agile execution of the management strategies.

Project Size. The larger the project (as represented by "the dollars spent", "the size of the implementation staff", "the time allocated to its implementation", and "the number of organizational units affected"), the higher likelihood of risk realization. However, if a company' has had a lot experience with projects exceeding a given size, then a new project of about the same size has less likelihood of risk realization.

Project Structure. Some projects are more highly structured than others and their requirements are clear and straightforward, so that the outputs and processes can be easily defined. Users know exactly what they want and what the system should do; there is hardly any possibility of their changing their minds. Such projects have much lower risks than those whose requirements are relatively undefined, fluid, and constantly changing, where outputs cannot be easily defined because they are subject to users' changing demands or because users cannot agree on what they want.

Experience with Technology. The project risk will rise if the project team and the information system staff lack the required technical expertise. If the team is unfamiliar with the hardware, the system software, the application software, or the database management system proposed for the project, it is highly likely that one or all of the following will occur: An unanticipated failure to deliver by the due date because of the need to master new skills; A variety of technical problems if tools have not been thoroughly mastered; Excessive expenditures and extra time because of inexperience with the undocumented idiosyncrasies of unfamiliar pieces of hardware or software.
Risk evasion strategy: System structuring can reduce risks accompanying system development. Technologically, system structuring can be effectively promoted by standardization of hardware protocol (open system) and employment of a combination of structured analysis / design and the DOA (data-oriented approach) for the development of application systems. In relation to the human and organizational aspect of the problem, effective means of risk reduction include enhancement of engineers' experience and skill, promotion of educational training of users, reduction of workload (engineers' overload), job enrichment, utilization of external consultants, etc.

Evaluation: This aspect of flexibility should be evaluated in terms of the easiness of changing or adding new application functions to an existing MIS. This easiness is constrained by the degree to which an existing MIS made up of hardware, application systems, and network is structured. However, since hardware and network can be evaluated in terms of the flexibility factor of "exchangeability" if they share the standardized communication protocol, here we will focus on the evaluation of application systems.

The ease of changing application systems is a function of the degree to which they are structured. Therefore the $P O C$ for the evasion strategies needs to be estimated with a focus on the structuring of application systems, programs and data. In the evaluation of data structure, fundamental indices are the number of access paths from an application program to the data and the number of programs and data items that need to be changed. A suitable criterion for evaluation of the whole data structure of an MIS is the ratio of entities incorporated into the database to the whole entities for management in the BP (business process) (i.e. whether the data is normalized and a data dictionary is provided). The utility of database incorporation can be estimated in terms of the reduction of the $P O C$ compared with the potential $P O C$ to be incurred where databanking is not implemented.

The $P O C$ here is cost and time required to cope with change demands, and should be evaluated by estimating each system alternative, which is a combination of a variety of hardware, application systems and network. The $P O C$ and utility of risk evasion strategies can be evaluated by estimating cost and time for the strategies. In economic evaluation, it is necessary to compare the difference in the cost and utility of system implementation, which depend on whether the system is structured or not. In addition, quantitative monitoring of backlogs will provide useful 


\section{Database Agile Management Dependent on MIS Infrastructure}

information when judging system implementation in terms of this index of flexibility.

Relationship with external factors: A well-structured application system can easily provide a "product" that will satisfy the requirements for an MIS function to be provided and this in the form of a combination of components of an existing MIS. It can therefore reduce the "volume" of the program to be newly developed or modified. Moreover, enhancement of the flexibility factor of "volume" enhances the flexibility of "working sequence". The internal flexibility factor of "system structure" in effect contributes to the enhancement of the external flexibility factors of "product", "volume" and "working sequence".

Example: Data independency will make it possible to carry out a change of a data processing procedure only by a change of programmed logic. A well-structured program will make it possible to change a data processing procedure by a mere change of the modules concerned, e.g. modules of the program constituting the subsystem concerned or by definition change in DD (see Case 1). A wellstructured system will also enable partial implementation of an MIS function in keeping with the proficiency level of the user (see Case 2).

\section{Service Area}

Definition: The ability to provide a service in an unfamiliar or new area.

Cause of change: System development concerning an unfamiliar or new service area.

Risk of change: Lack of experience generates risks. Risks such as failure to deliver by the due date, malfunctioning, and system failure are built into an MIS in the process of system development.

System development projects differ remarkably in difficulty depending on their size, scope, level of complexity, and organizational and technical factors. Some system development projects are more likely to fail because they contain a much higher level of risks than others. Researchers have identified three key dimensions, Project Size, Project Scope, Experience with Technology, which affect the level of project risks (McFalan, 1981) (see Table 3). These dimensions of project risks will be present in different combinations for different implementation efforts.
Moreover, of no less importance than the above three dimensions is the timing of a system development or the environment in which it is undertaken. An organization that has been used to long years of stability is apt to show a strong resistance against a change. In such a circumstance, it is difficult to acquire the support of the organization concerned and resources required for the system development. Often large-scale system development projects are undertaken at a time of an organizational crisis or rather to cope with the crisis. Maximum urgency of such a project will not allow of adequate and thorough planning. In neither of the cases is it advisable to implement a large-scale system development.

Risk evasion strategy: The basics of risk evasion, however, lie in paying attention to project scale, project structure, technological experience, and the timing of a system development. These depend on experience. Figure 2 shows entities for management in a familiar and a new and unfamiliar service area. Since Entity-A has already been implemented into the database for Served Area, its nature is familiar at the time of system design for New Area. In order to define the characteristics of an unfamiliar area we need to list all the entities for management in the BP of the enterprise concerned. This purpose would be best served by the application of the DOA. In the development of an application system for a new BP (to which MIS service has not been provided), if an entity (included in a new BP) is also an element of the other served BP, the labor of system design for this new BP will be reduced as far as this entity is concerned, since incorporation of the entity into the database is a known quantity. Accordingly, listing of all the entities for management beforehand will be useful for the estimation of risks we know to be inevitable in system development for an area that demands change.

Evaluation: Entities for management in an enterprise sel-

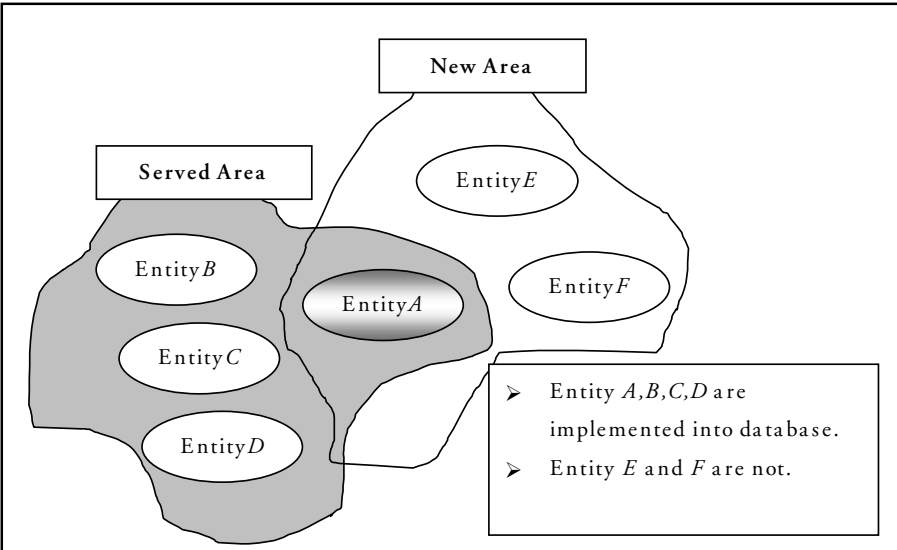

Figure 2. Entity in Unfamiliar Service Area 
dom undergo any fundamental change unless there occurs a change in industry type. This means that a change of a business function procedure hardly affects the data structure of the system built on a DOA basis. If service target entities include service targets in a familiar area, these can be expected to remain intact in terms of data structure. Thus, man-months needed for system design will be limited to labor involving new service target entities (Martin, 1989).

Therefore, in a comprehensive evaluation of an existing MIS, it would be reasonable to focus on the ratio of service target entities to the whole entities under the management of the organization concerned. As for a new service area, the focus of evaluation will be on the degree to which service target entities in the new area include service targets in the familiar area.

In the evaluation of $P O C$ in respect of this category, we need to estimate the $P O C$ for each system alternative as in the case of the category of "system structure". Since entities for management are stable, system design needs to pay the $P O C$ only once. But the utility of the design will remain effective in the future system development.

Economic utility can be evaluated in terms of the reduction of the $P O C$ (reduction of man-months for system design) due to the partial dispensability of labor for system design in the unfamiliar area.

Relationship with external factors: A high degree of experience in a target "service area" facilitates "product" design. Likewise the listing of entities for management will enhance the flexibility of "product" in the sense that it will contribute to the satisfaction of the requirements for the function to be provided. The reduction of labor for system design will enhance the flexibility of "volume", which in turn will enhance the flexibility of "working sequence".

\section{IT Adoption}

Definition: The ability to provide service using unfamiliar or new technology and/or development methods.

Cause of change: Technological innovation planned by an MIS division: i.e. exchange of computers and network due to technological innovation (e.g. downsizing) or system development by means of new techniques.

Risk of change: As with the category of "service area", risks are generated by lack of experience. But risks of this kind are dissolved by accumulation of experience and pro- ficiency in the use of new technology, which is likely to increase to some extent with the passage of time.

Risk evasion strategy: Since risks are engendered by mistakes in design, deficiency of engineers' experience and skill, inadequate education and training for users, there is a need for lead-time for implementation of new technology to be spent for its mastery. It is advisable to avoid introducing new technology into a project whose due date is urgent, since urgency aggravates potential risks. Other effective risk evasion strategies are $\mathrm{R} \& \mathrm{D}$, standardization of development methods, educational training (dissolution of skill deficiency).

Evaluation: The continuity between new technology and technology that has been used in an existing MIS greatly affects the time it will take to learn new skills in using it. The focus of evaluation needs to be on the degree of proficiency. Inadequate proficiency can even lower productivity when new technology is used. In the evaluation of this flexibility factor, the evaluation of $P O C$ must be based on an estimate of every system alternative, as with the category of "system structure". Since risk evasion strategies are limited to technological learning, the $P O C$ must be estimated in terms of proficiency growth on the time axis. The greater the degree of proficiency, the smaller is the $P O C$ that will have to be paid (see Figure 3).

\section{Relationship with ex- ternal factors: The} degree of learning on new technology enhances (or lowers) the facility of realizing a "product" such that it

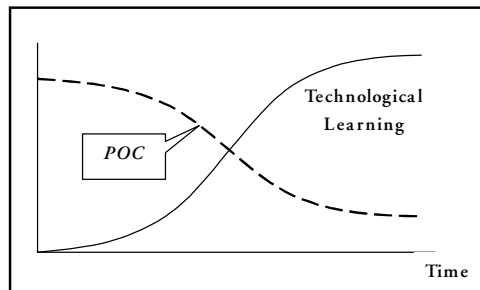

Figure 3. Technological Learning and $P O C$. will satisfy the requirements for the MIS function. The increase (or decrease) of man-months for system design due to the degree of proficiency affects the flexibility factor of "volume" positively or negatively., which in turn affects the flexibility factor of "working sequence" in a similar way.

Example: In early 90's, since downsizing was the mainstream of MIS development, the Grosh's law, which states that "capacity of a computer depends on the price of its CPU (central processing unit) power" ceased to make any sense. Not only that. Technological continuity, which allpurpose computer manufacturers had been providing, was also wiped out. All this led to frequent occurrence of troubles involving both system development and system use 


\section{Database Agile Management Dependent on MIS Infrastructure}

before engineers managed to master heterogeneous technology. We could hardly say that technological innovation engendered higher MIS flexibility (See Case 3).

\section{Relationship between Internal and External Fac- tors}

\section{Case 3: Downsizing as an Implementation of New Technology}

Printing paper container manufacturer $\mathrm{Z}$ had used on a mainframe a fairly sophisticated system for scheduling and control of production targeted at printing and subsequent processes. In 1994, with its stocks due to go public the next year (an organizational crisis), the manufacturer decided to build a sales management system. In those days downsizing was the fashion of the day in Japan. Jumping on the bandwagon, this company decided to build the system on client/server architecture (which was an utterly new technology discontinuous with the previous technology). The system development, accompanied by a purchase of PCs for development (They intended to use them in their routine work on completion of the new development), was outsourced to a vendor. The development of this system on this basis took far less time than on a mainframe. Adopting a prototyping-like development approach and using a relational DBMS, they had a user-friendly application system completed one year later.

Unfortunately, however, the capacity of the one-year-old PCs was less than sufficient to let this system work and replacing them with the latest high-end PCs required extra cost. Furthermore, upgrading to a new OS for the PCs required a great deal more extra cost for lack of upper compatibility. For several years after that, the company found to their great disappointment that with the PC-based system they had to cope with far more system failures and their recoveries than with the mainframe-based system.

Several years later, they undertook another change, this time in the system for data communication with customers, but the change took far more time and labor than they had expected due to deficiency in documentation. Their choice of technology was the trend of the times. But the heterogeneous new technology that they had adopted continued to make fun of the engineers. Now the heterogeneous monster has been tamed and become relatively obedient. In other words, the new technology has become more flexible.

Generally implementation of new technology entails the high risk of system failures (i.e. an information system that either won't perform as expected, is not operational at a specified time, or cannot be used in the way it is intended to be). Successful implementation does not mean an immediate realization of effectiveness because it takes time for the users to acquire proficiency in the use of the new system. System trouble obstructs flexible use of an MIS. A project like this whose due date is critical requires a high degree of MIS flexibility (IT adoption).

In this case, to obtain the same MIS function, it would have been more advantageous if they had modified the existing MIS working on mainframe because it would have incurred a far smaller $P O C$ than did the introduction of new technology.
The relationship between internal and external factors is as follows:

The flexibility of "product" (Flex(pd)) as an external factor is constrained by the internal factors of "exchangeability" $($ Flex(ex)) and "system structure" (Flex(st)).

$$
\text { Flex }(p d)=f_{p}(\text { Flex }(\text { ex }), \text { Flex }(\text { st }))
$$

The flexibility of "volume" (Flex $(v l))$ as an external factor is constrained by the internal factors of "fault tolerance" (Flex(tl)), "system structure", "service area"(Flex(ar)) and "IT adoption" (Flex(IT)).

$$
\begin{array}{r}
F \operatorname{ex}(v l)=f_{v}(F \operatorname{lex}(t l), \text { Flex }(s t), \\
F \operatorname{ex}(\operatorname{ar}), F \operatorname{ex}(I T))
\end{array}
$$

The flexibility of "working sequence" (Flex(sq)) as an external factor is constrained by the external factor of "volume".

$$
\operatorname{Flex}(s q)=f_{s}(F l e x(v l))
$$

Thus the flexibility of a whole MIS can be formulaically represented as follows:

$$
\begin{gathered}
\text { Flex }(M I S)=f(F l e x(p d), F l e x(v l), F l e x(s q)) \\
=g(\text { Flex }(\operatorname{ex}), F l e x(t l), F l e x(s t), \\
\text { Flex }(\text { ar }), \text { Flex }(I T))
\end{gathered}
$$

That is to say, renovation of MIS infrastructure is nothing other than the flexibility enhancement of the internal factors of "fault tolerance", "system structure", "service area", and "IT adoption".

Here let us recall formula (3), in relation to which strategies for the renovation of MIS infrastructure were discussed in terms of risk evasion strategies.

\section{Scheme for Evaluation Procedure of MIS Flexibility}

Let us note that the first step in strategic management is to decide on several goals and that the next step is to work out strategies to attain these goals. MIS flexibility needs to be just sufficient to accomplish these strategies and this much flexibility must be secured in advance. Therefore we propose that MIS planning should be executed according to the following procedure. To begin with, we will predict 
future demands for changes likely to be made on an MIS and through the other three steps we will consider how to provide against these change demands (see Figure 4).

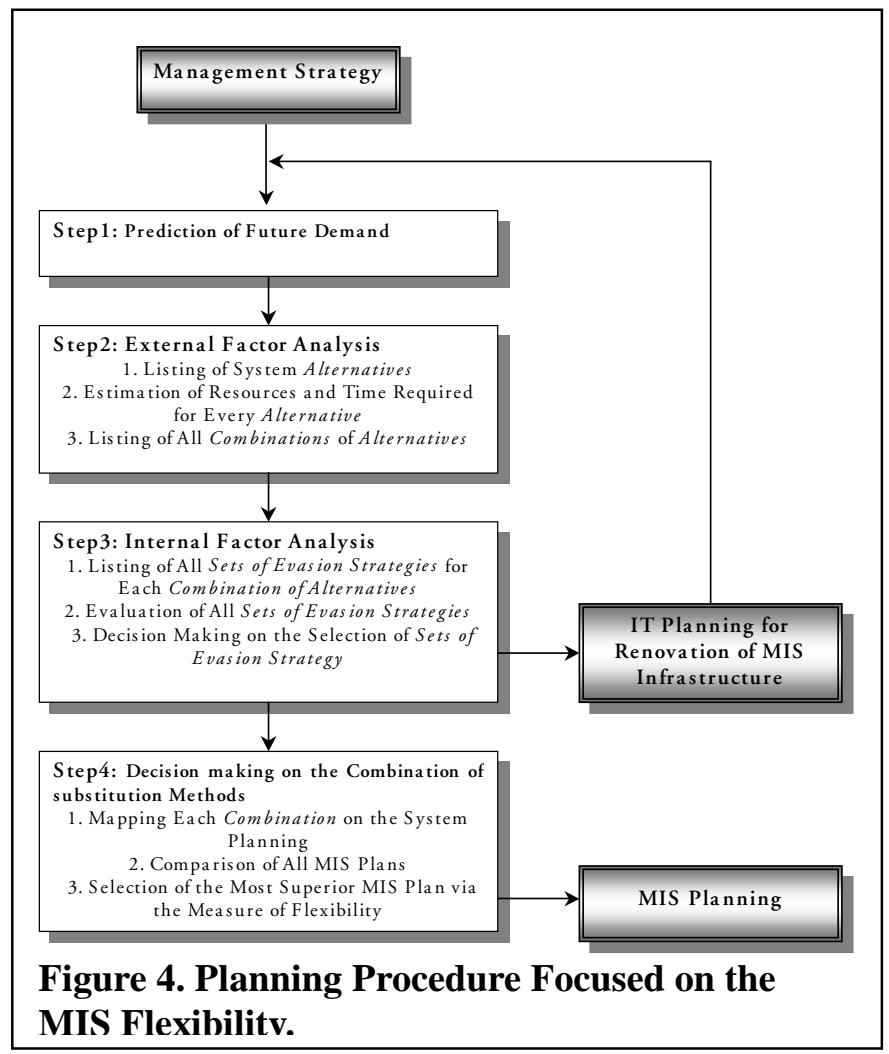

\section{Step 1: Prediction of Future Demands}

Our task in this step is to predict change demands likely to be made on an MIS originating from management strategies, user needs and IT renovation planning by the MIS division.

\section{Step 2: External Factor Analysis}

The first part of this step is devoted to extracting as many candidate system alternatives as possible (for system implementation) to deal with all change demands predicted. The questions for us to ask in doing the above are:

- What kinds of system alternatives are applicable to accomplish this MIS change? (Product),

- What amounts of resources will a combination of system alternatives require in the development of this system and in its use process? (Volume),

- Does the existing MIS infrastructure have enough tolerance for absorption of operation order changes when an unexpected disturbance (a change demand on the
MIS and/or a system breakdown) occurs? (Working sequence).

Our work in the rest of this step is to estimate the required resources for each system alternative based on the present condition of the system and the ITs we have available. Since multiple change demands predicted must be disposed of, the last thing to do here is to enumerate combinations of system alternatives to be used.

\section{Step 3: Internal Factor Analysis}

This step addresses the question of how to enhance internal MIS flexibility in order to maximize the efficiency in the disposal of change demands. We will first enumerate, for each combination of system alternatives, future system risks, their probability of occurrence and strategies for their evasion (e.g. method for system structuring and for normalizing data). See Table 1 . Next we will evaluate the efficiency of these risk evasion strategies and after studying their feasibility, we will enumerate several sets of evasion strategies (strategies for infrastructure renovation) to be applied to each of the combinations of system alternatives. We must note here that these evasion strategies themselves generate new change demands in the form of "IT plans for MIS flexibility enhancement" as shown in Figure 4.

\section{Step 4: Decision making on the Combinations of System Alternatives}

With all the factors required for MIS planning provided as in the above three steps, our next step is to map each combination of system alternatives onto MIS planning. By

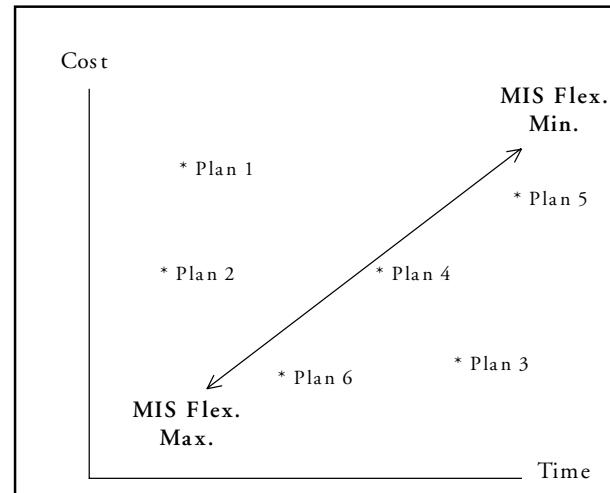

Figure 5. Mapped MIS Plans. comparing the mapped combinations with each other, we will be able to identify a combination with maximum flexibility as in Figure 5. 


\section{Database Agile Management Dependent on MIS Infrastructure}

\section{Conclusion}

In this paper, we have focused on and presented the following: the causes of change demands on an MIS, the internal and ex external factors that an MIS should maintain to cope with change demands, and the procedure for evaluation of MIS flexibility via analysis of the relationship between the internal and external factors.

Specifically, we have elucidated the following:

- MIS flexibility is an index of the ability of an MIS to absorb future change demands on it.

- MIS flexibility can be evaluated quantitatively in terms of the substitute index of $P O C$ (penalty of change).

- MIS flexibility consists of internal and external factors. Evaluation of external factors focuses on the volume of change demands an MIS can absorb, and evaluation of internal factors focuses on the variety of strategies to evade change risks. We have treated $P O C$ as a common index to evaluate the two categories of MIS flexibility factors.

- External factors of MIS flexibility are constrained by internal factors.

- Renovation of MIS infrastructure means application of information technology for evasion of change risks accompanying MIS implementation.

- Proper renovation of MIS infrastructure will enhance the internal characteristics of MIS flexibility.

The challenges confronting us ahead are the detailed and practical studies on the following:

- quantitative relationship between each internal and external factor,

- behavior of cost and utility of MIS use and MIS infrastructure on the time axis.

\section{References}

Brooks Frederick, P., (1974). "The Mythical Man Month, "Datamation, Vol.20, No.12, pp.44-52.

DeLone, William H, and Ephraim R. McLean, (1992). "Information System Success: "The Quest for the Dependent Variable", Information Systems Research, Vol.3, No.1, pp.60-95.

Johanson, H.J., McHugh, P., Pendlebury, A.J. and Wheeler, W. A., (1993). Business Process reengineering: break point strategies for market dominance, Willey, p.4.

Hamilton, S. and Chervany, N. C, (1981). "Evaluating Information System Effectiveness - Part 1: Comparing Evaluation Approaches", MIS Quarterly, pp.55-69.
Hanaoka, S., (1997). "Relevance between Strategic Alliance and Characteristic of Competitive Core", Journal of the Japan Society for Management Information, Vol.6, No.1, pp.69-86.

Laudon, K. C. and Laudon J.P., (1994). Management Information Systems: Organization and Technology, 3rd Ed., Macmillan College Publishing Company Inc.

Laudon, K. C. and Laudon J.P.: Management Information Systems: Organization and Technology in the Networked Enterprise, 6rd Ed., Prentice-Hall, Inc., 2000.

Lucas, Henry C., Jr., (1974). Toward Creative Systems Design, New York: Columbia University Press.

Martin, J. and Leben, J., (1989). Strategic Information Planning Methodologies 2nd Ed., Prentice Hall, Inc.

McFarlan, F. Warren, (1981). "Portfolio Approach to Information Systems," Harvard Business Review, Vol.59 No.5, pp.142-150.

Myer, N. Dean and Boone, Mary E., (1989). The Information Edge, The Carswell Company.

Parker, M.M. and Benson, R.J., (1988). Information Economics: Linking Business Performance to Information Technology, Prentice Hall, Inc..

Pnuell, Y. and Zussman, E., (1997). "Evaluating the end-of-life value of a product and improving it by redesign", International Journal of Production Research, pp.921-942.

Richardson, P. R. and Gordon, J. R. M., (1980). "Measuring Total Manufacturing Performance", Sloan Management Review, Vol.9, No.2, pp.47-58.

Users Guide for Information System (I), (1981). Japan Information Processing Development Center, (in Japanese).

Utunomiya H., Ohashi H.,Takahashi I. and Miyagawa Y., (1993). "The Evaluation of Information Systems", Journal of Information Processing Society of Japan, 93-IS-46, Vol.96, No.90, pp.1-40, Information Processing Society of Japan, (in Japanese).

Yin, Robert K., (1981). "Life Histories of Innovations: How New Practices Become Routinized," Public Administration Review, pp.2128 .

\section{Biographies}

Masaru Furukawa is a faculty member of TOYAMA University in Japan, where he teaches management information systems. He has ten years of practical experience as a manager of MIS division in a large enterprise and seven years of teaching experience as a Professor. 
For edition of the manuscript for its English, thanks are owed to Akira Minami, a Professor of English at Toyama Prefectural University, Japan. 